\title{
Journal of Translational Medicine reviewer acknowledgement 2015
}

Francesco Marincola*

\section{Reviewer acknowledgement}

The Editors of Journal of Translational Medicine would like to thank all our reviewers who have contributed to the journal in Volume 13 (2015).

Sayed Abdelwahab
Egypt
Ludovico Abenavoli
Italy

Jg Abreu

Brazil

Raffaele Addeo

Italy

Adetola Adesida

Canada

Sagheer Ahmed

Brunei

Wk Aicher

Germany

Laura Alaniz

Argentina

Donatella Aldinucci

Italy

Edoardo Alesse

Italy

H. Richard Alexander

USA

Jean-Pierre Allain

UK

Ahmed Al-Qahtani

Saudi Arabia
Frédéric Amant

Belgium

Ryszard Amarowicz

Poland

Muriel Amathonnet

France

Aditya Ambade

USA

Guoy An

China

\section{Anuranjan Anand}

India

Julian Ananiev

Bulgaria

Sarah Andres

USA

Polychronis Antonitsis

Greece

Vivekanandhan Aravindhan

India

Thurayya Arayssi

Qatar

\section{Adria Arboix}

Spain

Mario Arciello

Italy

\author{
Andressa Ardiani \\ USA \\ David Argyle \\ UK \\ Elif Damla Arisan \\ Turkey
}
Abdelilah Arredouani
Qatar
Inesa Arstikyte
Lithuania

Anders Asberg

Norway

Hitoshi Ashida

Japan

Stephanie Astrow

USA

Djordje Atanackovic

Germany

Djordje Atanackovic

USA

Stephen Atkin

Qatar

David Avigan

USA

Julio Ayala

USA

*Correspondence: jtransmed@biomedcentral.com

Sidra Medical and Research Center, Doha, Qatar 
Angel Ayuso-Sacido

Spain

Ricardo Azziz

USA

Biersack B

Germany

Lorena Baccaglini

USA

Christina Bade-Doeding

Germany

Radja Badji

Qatar

Mohammed Badruddoja

USA

Peter Bai

Hungary

Salar Bakhtiyari

Iran

Ligia Bancu

Romania

Sanghamitra Bandyopadhyay

India

Barbara Barbaro

Italy

Maria Marcela Barrio

Argentina

Shashi Baruah

India

Giuseppina Basini

Italy

Purusotam Basnet

Norway

C.N. Baxevanis

Greece

Zeliha Bayram

Turkey

Fuller Bazer

USA

Nicole Beauchemin

Canada

George Beck

USA
Nicolau Beckmann

Switzerland

Diana Bell

USA

Giuseppe Bellisola

Italy

Gavin Bendle

The Netherlands

Armand Bensussan

France

Michael Bergmann

Austria

Catherine Berthomieu

France

Antonio Bertoletti

Singapore

Nicholas Bertos

Canada

Pascale Bertrand

France

Ricardo V Bessa-Nogueira

Brazil

Ali Bettaieb

France

Avinash Bhandoola

USA

Anshu Bhardwaj

India

Deepa Bhartiya

India

Karen Bieback

Germany

Carlo Bifulco

USA

Catherine Bisbal

France

Priscilla Biswas

Italy

Lena Björkman

Sweden

Julia Blanco

Spain
Jeronimo Blanco

Spain

Michael Blennerhassett

Canada

Javier Blesa

Spain

Erik Blok

The Netherlands

Edimar Bocchi

Brazil

Manfred Boehm

USA

Martin Bonamino

Brazil

R. Daniel Bonfil

USA

William Bonner

USA

Sahra Borges

USA

Laszlo Boros

Hungary

Francisco Borrego

Spain

Emanuela Bostjancic

Slovenia

Hamid Boulares

USA

Claudio Brancolini

Italy

Timo Brandenburger

Germany

Caterina Brasacchio

Italy

Gary Braun

USA

Caroline Breitbach

USA

Guilherme Bresciani

Chile

Nicola Brown

Australia 


\author{
Joseph Bryant \\ USA \\ Ulrike Buchwald \\ USA \\ Alfredo Budillon \\ Italy \\ Linda Burkly \\ USA \\ Gregory Calip \\ USA
}

Sidia M. Callegari-Jacques

Brazil

Rafael Camargo

Brazil

Mark Cameron

USA

Robert Canter

USA

Jiatian Cao

China

Wei Cao

China

Haiming Cao

USA

Sergio Capaccioli

Italy

Emilia Caputo

Italy

Michele Caraglia

Italy

Nora Cardona-Castro

Colombia

Gloria Patricia Cardona-Gomez

Colombia

\section{Anna Caretti \\ Italy \\ Ivana Carey \\ UK}

Matteo Carlino

Australia

Tom $_{i}$ Carroll

Ireland
Dennis Carson

USA

Wayne Carver

USA

Luciano Castiello

Italy

Luciano Castiello

Italy

Patricia Castro Santos

Spain

Georgescu Ce

Romania

David Chafin

USA

Antonio Carlos Chagas

Brazil

Roger Chammas

Brazil

Stephen Chan

USA

Kw Chan

Hong Kong

Hsueh-Wei Chang

Taiwan

Wen-Wei Chang

Taiwan

Christopher Chang

USA

Jehad Charo

Germany

Tapas Kumar Chaudhuri India

Damien Chaussabel

Qatar

Novel N. Chegou

South Africa

Fa-Ming Chen

China

Chunyan Chen

China

Chuan Chen

China
Hui Chen

China

Junqiang Chen

China

Lijuan Chen

China

Fuxue Chen

China

Bor-Sen Chen

Taiwan

Mei-Lien Chen

Taiwan

Zongming Eric Chen

USA

Chu-Huang Chen

USA

John Chen

USA

Ye Chen

China

Hao Cheng

China

Xi Cheng

China

Alfred Cheng

Hong Kong

Zhijie Jey Cheng

USA

Sung-Gil Chi

South Korea

Ya-Hui Chi

Taiwan

Maurizio Chiriva-Internati

USA

Miguel Chiurillo

Venezuela

Mi-La Cho

Iammila@Catholic.Ac.Kr

Mi-La Cho

South Korea

Dong-Sic Choi

South Korea 
Murim Choi

South Korea

Eun Young Choi

South Korea

Mukund Chorghade

USA

Mukund Chorghade

USA

Becky Christian

USA

Georgios Christou

Greece

George Chrousos

Greece

Ji Chuang

Taiwan, China

June-Key Chung

South Korea

Christine Chung

USA

Dana Mihaela Ciobanu

Romania

Shiyuan Cjeng

USA

William Coleman

USA

Jucimara Colombo

Brazil

Begonya Comin-Anduix

USA

Angelo Giuseppe Condorelli Italy

Jose Conejo-Garcia

USA

Costanza Conti

Italy

Leonel Cordoba

Costa Rica

Robin Cornelissen

The Netherlands

Andrea Cossarizza

Italy
Alexessander Couto Alves

UK

Johannes Coy

Germany

David Cram

China

Dragos Cretoiu

Romania

Lucio Crino'

Italy

Kylie Crompton

Australia

Bla $_{i}$ Cugmas

Slovenia

Chiara Cugno

Qatar

William Culp

USA

Giuseppe Curigliano

Italy

Brian Czerniecki

USA

Nayle Da Silva

Brazil

Agustin Dalmasso

USA

Bruno Daniele

Italy

Gennaro Daniele

Italy

Fabrice Danjou

France

Fida Dankar

Qatar

Nazir Dar

India

Piyali Dasgupta

USA

Jean Pascal De Bandt

France

Tjaart De Beer

Switzerland
Jana De Boniface

Sweden

Laura De Girolamo

Italy

Andrea De Maria

Italy

Valli De Re

Italy

Anita De Rossi

Italy

A. De Siervi

Argentina

Roel De Weger

The Netherlands

Olivier De Wever

Belgium

Julie Decock

Qatar

Maureen Deehan

Switzerland

Nuria Del Olmo

Spain

Bahman Delalat

Australia

Colleen Delaney

USA

Christian Delles

UK

Yanru Deng

USA

Melissa Derycke

USA

Sophie Desplat-Jégo

France

Massimo Di Maio

Italy

Stefania Di Marco

Italy

Laura Di Renzo

Italy

Sandra Diebold

UK 
Michelle Digiacomo

Australia

Xiaoqiang Ding

China

Shaillay Dogra

Singapore

Kaoru Dohi

Japan

Riccardo Dolcetti

Italy

Helmut Dolznig

Austria

Pere Domingo

Spain

Shiwu Dong

China

Peixin Dong

Japan

Gina Doody

UK

Thilo Dork

Germany

Simon Dovedi

Norman Dovichi

USA

Hans C. Dringenberg

Canada

Jaroslav Drsata

Czech Republic

Jun Du

China

Alfonso Duenas-Gonzalez

USA

Hélène Dumortier

France

Greg Dusting

Australia

Almac E.

USA

Sancak Eb.

Turkey
M. Eberl

UK

Jose Echevarria

Spain

Gunter Eckert

Germany

Ihab El Madhoun

Qatar

Bernice Elger

Switzerland

Hassan El-Sayyad

Egypt

N. Elsherbini

Egypt

Edward Emmott

UK

Elena Enache

Romania

Anna-Mart Engelbrecht

South Africa

Perenlei Enkhbaatar

USA

Amalia Enriquez-De-Salamanca

Spain

Alan Epstein

USA

Philipp Erben

Germany

Neta Erez

Israel

Mark Erlander

USA

Galileo Escobedo

Mexico

Xavier Estivill

Spain

Fabio Fabbian

Italy

Chiara Fabbro

Italy

Astrid V. Fahlenkamp

Germany
Birthe Fahrenkrog

Belgium

Stefano Fais

Italy

Domenick Falcone

USA

Xiaolong Fan

Sweden

Arghavan Farzadi

Malaysia

Todd Fehniger

USA

Ariel Feldstein

USA

Jane Ferguson

USA

David Ferland-Mccollough

UK

Cristiano Ferlini

USA

Silvia Martina Ferrari

Italy

Ludovic Ferretti

France

Clodoveo Ferri

Italy

Robert Ferris

USA

Fernando Fierro

USA

Fabiana Filigheddu

Italy

Brian Finck

USA

Ugo Fiocco

Italy

Andre Fischer

Germany

Timothy Fitzgerald

USA

Willy Flegel

USA 


\begin{tabular}{|c|c|c|}
\hline Megan Foeller & Mayuko Furuta & Ruowen Ge \\
\hline USA & Japan & Singapore \\
\hline Valentina Folgiero & Hala Gabr & Joel Gelfand \\
\hline Italy & Egypt & USA \\
\hline Joseph Fontes & Giovanni Gaeta & Panagiotis Georgianos \\
\hline USA & Italy & Greece \\
\hline Brian Ford & Bertino Gaetano & Hanspeter Gerber \\
\hline UK & Italy & USA \\
\hline Claudio Fozza & Deni Galileo & Amato Giaccia \\
\hline Italy & USA & USA \\
\hline Gail Fraizer & Martha Patricia Gallegos & Luca Giacomelli \\
\hline USA & Mexico & Italy \\
\hline Renato Franco & Grazia Galleri & Clemens Giessen \\
\hline Italy & Italy & Germany \\
\hline Carlos Franco-Pparedes & Andrea Galli & Carla Giordano \\
\hline USA & Italy & Italy \\
\hline Vanessa Morais Freitas & Gary Edward Gallick & Giorgia Girotto \\
\hline Brazil & USA & Italy \\
\hline Marilia Freitas Calmon & Maria Elsa Gambuzza & Rebecca Gladdy \\
\hline Brazil & Italy & Canada \\
\hline Gregory K. Friedman & Nalini Ganesan & W. Scott Goebel \\
\hline USA & India & USA \\
\hline Jan Frystyk & Can Gao & Sucheta Gokhale \\
\hline Denmark & China & India \\
\hline Jiao Fu & Chongfeng Gao & Diego Golombek \\
\hline China & USA & Argentina \\
\hline Yang-Xin Fu & Spiros D. Garbis & Ping Gong \\
\hline USA & UK & China \\
\hline Dietmar Fuchs & Helena M. Garcia & Ramakrishnan Gopalakrishnan \\
\hline Austria & Portugal & USA \\
\hline Maria Fuciarelli & Iraia García & Patricia Grabowski \\
\hline Italy & Spain & Germany \\
\hline Shin-Ichiro Fujii & Mariana Haydee García Hernández & Stephan Grage \\
\hline Japan & Mexico & Germany \\
\hline Shigetomo Fukuhara & Marisa Gariglio & Jennifer Granick \\
\hline Japan & Italy & USA \\
\hline Valerio Fulci & Charlie Garnett-Benson & Marco Grasso \\
\hline Italy & USA & Italy \\
\hline Nicholas Funderburg & Andrei Gartel & Francesca Grati \\
\hline USA & USA & Italy \\
\hline Nicholas Funderburg & Luca Gattinoni & Tim Greten \\
\hline USA & USA & USA \\
\hline
\end{tabular}


Jörg Großhans

Germany

Viktor Grünwald

Germany

Congying Gu

USA

Jian Guan

China

Andrea Guennoun

Germany

Andrea Guennoun

Qatar

Pokhraj Guha

India

Maria Valle Guijarro

USA

Hongqian Guob

USA

Rakesh Gupta

India

Raphael Guzman

Switzerland

Duy Ha

USA

Patrick Ha

USA

Ramsey Hachem

USA

Abigail Hackam

USA

Michael Hackenberg

Spain

Monika Haemmerle

USA

Weidong Han

China

Patrick Hanley

USA

Joshua Hare

USA

Ulrike Harre

Germany
Lauren Harshman

USA

Akira Hashiramoto

Japan

Larisa Haupt

Australia

Yong He

China

Nongyue He

China

Gen He

China

Xiangjun He

China

Meian He

China

Lionel Hebbard

Australia

Doreen Heckmann-Nötzel

Germany

Sean Heffron

USA

Shelly Heimfeld

USA

Mahasti Hemanth

India

Peiman Hematti

USA

Alonso Heredia

USA

Caroline Herron

Ireland

Em Higa

Brazil

Henry Higgs

USA

Jason Hinman

USA

Christoph Hoeller

Austria

L. John Hoffer

Canada
Marion Hofmann-Bowman

USA

Stefan Holdenrieder

Germany

Arne Homann

Germany

Huang Hongbiao

China

Risto Honkanen

Finland

Xuwei Hou

China

Liwen $\mathrm{Hu}$

China

Wanchung $\mathrm{Hu}$

Taiwan

Guo-Fu Hu

USA

Xiaojun Huang

China

Chen Huang

China

Kuo-How Huang

Taiwan

Michael Hudecek

Germany

Petra Hudler

Slovenia

Kam Hui

Singapore

Esther Hulleman

The Netherlands

Amanda Hummon

USA

Bonnie Hylander

USA

Tommaso Iannitti

UK

Thomas Ichim

USA

Giandomenica Iezzi

Switzerland 
Imran Imran

Germany

Giuseppe Intini

USA

Nida Iqbal

India

Demelza Ireland

Australia

Idilman Is

Turkey

Nobukazu Ishizaka

Japan

Anna Isinger Ekstrand

Sweden

Ayesha Ismail

India

Raphael Isokpehi

USA

Tomer Itkin

Israel

Sawa Ito

USA

Keisuke Ito

USA

Takahashi J.

Japan

Li J.

China

Naranamangalam Jagannathan

India

Rubio Martinez Jaime

Spain

Andreas Janecke

Austria

Chaweewan Jansakul

Thailand

Jos Jansen

The Netherlands

Frank Jenkins

USA

Christie Jeon

USA
Mark Jeschke

Canada

Bente Jespersen

Denmark

Bin Ji

China

Chen Jiang

China

Lili Jiang

China

Yanfang Jiang

China

Kaiyu Jiang

USA

Pengfei Jiang

USA

Zhengyu Jiang

USA

Amjad Jilani

UK

Sergio Jimenez

USA

Ying Jin

China

Zhiming Jin

China

Ping Jin

USA

Puthen Jithesh

UK

Pascal David Johann

Germany

C. Hertzman Johansson

Sweden

Andrew Jones

UK

Aditya Joshi

USA

Oscar Juan

Spain

Park Jw

South Korea
Kazuhiro Kakimi

Japan

Michel Kalamarides

France

Kimberly Kalli

USA

Steve Kalloger

Canada

D. Kalvakolano

USA

Masakazu Kamata

USA

Nobuhiro Kanaji

Japan

Jia-Horng Kao

Taiwan

Mariana Kaplan

USA

Stylianos Karatapanis

Greece

Saoussen Karray

France

Mohammed Kashani-Sabet

USA

Hiroaki Kataoka

Japan

Rahul Kathariya

India

Johji Kato

Japan

Manabu Kawada

Japan

Melissa Kazantzis

USA

Andreas Keller

Germany

Gottfried Kellermann

USA

Christian Kellner

Germany

Philippe Kemoun

France 
Brian Kennedy

USA

Robert Kennedy

USA

Sid Kerkar

USA

Peter Kerkhof

The Netherlands

Santosh Kesari

USA

Ahmad Khalil

Mohammad Khan

Saudi Arabia

Mohsin Khan

USA

Mushfiquddin Khan

USA

Sameer Khandhar

USA

Tobias Kiesslich

Austria

Bg Kim

South Korea

Youngsoo Kim

South Korea

Sung-Hwan Kim

South Korea

Jang-Hee Kim

South Korea

Sung Kim

South Korea

Sh Kim

South Korea

Ju Kim

USA

Chan Hyuk Kim

USA

Gaspar Kitange

USA

Helmut Klocker

Austria
Joost Kluiver

The Netherlands

Jared Knickelbein

USA

Karl Richard Alexander Knuth

Qatar

Mario Kofler

Austria

Feliks Kogan

USA

Takashi Kohno

Japan

Navid Koleini

Canada

Tomas Konecny

USA

Gordana Konjevic

Serbia

Izabela Korczowska

Poland

Srigiridhar Kotamraju

India

Nir Krakauer

Oliver Holger Krämer

Germany

Stephanie Kreis

Luxembourg

Prasanna Krishnamurthy

USA

Karl Krueger

USA

Kostyantyn Krysan

USA

Dominika Ksiazek-Winiarek

Poland

Chia-Yi Kuan

USA

Selim Kuci

Germany

Ingo Kuhfuss

Germany
Joydeb Kumar Kundu

Bangladesh

Kv O'brien

USA

Peter Kwong

USA

Maris Laan

Estonia

Ahmed Labib

Qatar

Moncef Ladjimi

France

Brian Lane

USA

Philip Lang

Germany

Francesca Lantieri

Italy

Jacob Larkin

USA

Göran Larson

Sweden

Juan Jose Lasarte

Spain

George Lau

Hong Kong

Andrew Leask

Canada

Francis Y. Lee

USA

Hye Seung Lee

Hye2@Snu.Ac.Kr

Justine Lee

USA

Kyoo-Hyung Lee

South Korea

Hsin-Chen Lee

Taiwan

Linsheng Lei

China

Raya Leibowitz-Amit USA 


\begin{tabular}{|c|c|c|}
\hline Mathieu Lemaire & Chao-Hsiung Lin & Jiamiao Lu \\
\hline Canada & Taiwan & USA \\
\hline Gabor Lendvai & Li-Min Lin & Xiongbin Lu \\
\hline Hungary & Taiwan & USA \\
\hline Nibedita Lenka & Sue-Hwa Lin & Guilherme Lucas \\
\hline India & USA & Brazil \\
\hline Leticia Leon & David Lin & Tim Luckett \\
\hline Spain & USA & Australia \\
\hline Eleonora Leucci & Jere Linden & Jana Luetzkendorf \\
\hline Belgium & Finland & Germany \\
\hline Estrella Levy & Yinghui Ling & Enrico Lugli \\
\hline Argentina & China & Italy \\
\hline Alan Lewis & Lance Liotta & Miodrag Lukic \\
\hline USA & USA & Serbia \\
\hline Alan Lewis & Lei Liu & Ns Lurain \\
\hline USA & China & USA \\
\hline Zhijie Li & Song-Mei Liu & Hongjun Lv \\
\hline Australia & China & China \\
\hline Yan Li & Shaorong Liu & Anastasios Lymperopoulos \\
\hline China & USA & USA \\
\hline Pu Li & Zhaohui Liu & Duan Ma \\
\hline China & China & China \\
\hline Jinjun Li & Víctor Llombart & Daoxin Ma \\
\hline China & Spain & China \\
\hline Leping Li & David M. Loeb & Muzafar Ahmad Macha \\
\hline China & USA & USA \\
\hline Bing Li & Markus Loeffler & Catarina Madeira \\
\hline China & Germany & Portugal \\
\hline Zhong Li & Vincent Lombardi & Margaret Madeleine \\
\hline China & USA & USA \\
\hline Chenggang Li & Lucia Lopalco & Roberta Maggio \\
\hline China & Italy & Italy \\
\hline Zhe Li & Elena Lopez Villar & Karl-Eric Magnusson \\
\hline USA & Spain & Sweden \\
\hline Qing Kay Li & César López-Camarillo & Kathleen Mahoney \\
\hline USA & Mexico & USA \\
\hline Chih-Chuang Liaw & Antonio Lopez-Sanroman & Kristen Maitland \\
\hline Taiwan & Spain & USA \\
\hline Chuyong Lin & Marek Los & Eugene Major \\
\hline China & Sweden & USA \\
\hline Jian Lin & Jennifer Lowe & Joel Malek \\
\hline China & Brazil & Qatar \\
\hline
\end{tabular}




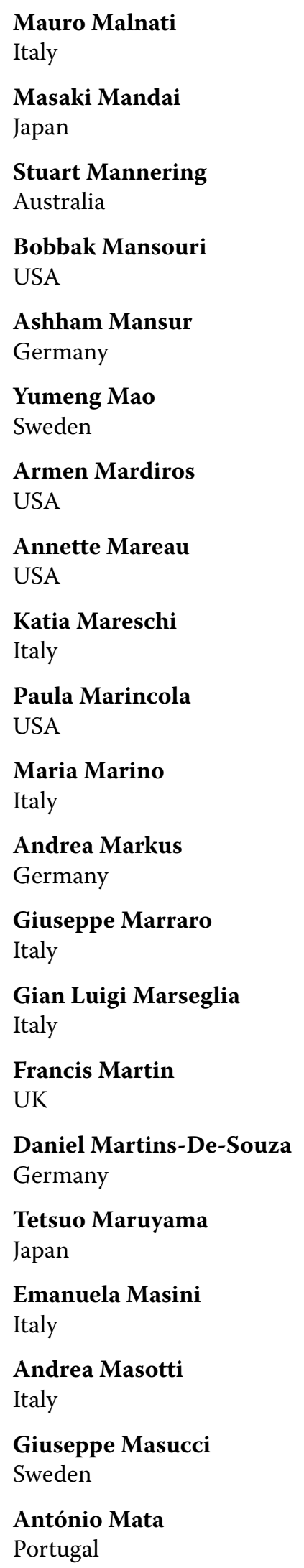

Jesús Mateos

Spain

Alicia Mathers

USA

Scot Matkovich

USA

Satoaki Matoba

Japan

Konstantinos Mavridis

Greece

Rose-Anne Mazure

Spain

Guillermo Mazzolini

Argentina

Kerrie Mcdonald

Australia

Donald Mcdonnell

USA

David Mckenna

USA

Randall Mckenna

USA

Radwa Mehanna

Egypt

Chitra Mehta

India

Shu Meng

China

Pier Luigi Meroni

Italy

Claudio Mesquita

Brazil

Patrick Meybohm

Germany

Thomas Meyer

Germany

Raposo Mf.

Portugal

Giancarlo Micheletto

Italy

Miguel Miguens

Spain
Michal Mikula

Poland

Humberto Milani

Brazil

Leslie Miller

USA

Wei-Ping Min

Canada

Silvia Minardi

USA

Boris Minev

USA

B. Minev

USA

Boris Minev

USA

Amadeo Minichino

Italy

Eva M. Mischak-Weissinger

Germany

Tom Misteli

USA

Ashim K. Mitra

USA

Tomomitsu Miyagaki

Japan

Yasuyoshi Miyata

Japan

Hiroshi Miyata

Japan

Alessandra Mocali

Italy

Marius Moga

Romania

Geetha Mohan

USA

Suyash Mohan

USA

Rajesh Mohanraj

United Arab Emirates

Giuseppe Montalto

Italy 


\begin{tabular}{|c|c|c|}
\hline $\begin{array}{l}\text { Bo-Hyun Moon } \\
\text { USA }\end{array}$ & $\begin{array}{l}\text { S. Nagini } \\
\text { India }\end{array}$ & $\begin{array}{l}\text { Gannon Np } \\
\text { USA }\end{array}$ \\
\hline $\begin{array}{l}\text { Martin Moore } \\
\text { USA }\end{array}$ & $\begin{array}{l}\text { Takayuki Nakagawa } \\
\text { Japan }\end{array}$ & $\begin{array}{l}\text { Heather M. O'hagan } \\
\text { USA }\end{array}$ \\
\hline $\begin{array}{l}\text { Ana Maria Mora } \\
\text { Costa Rica }\end{array}$ & $\begin{array}{l}\text { Yoshikazu Nakamura } \\
\text { Japan }\end{array}$ & $\begin{array}{l}\text { Natasa Obermajer } \\
\text { USA }\end{array}$ \\
\hline $\begin{array}{l}\text { Silvana Morello } \\
\text { Italy }\end{array}$ & $\begin{array}{l}\text { Kazuhiro Nakayama } \\
\text { Japan }\end{array}$ & $\begin{array}{l}\text { J O'callaghan } \\
\text { UK }\end{array}$ \\
\hline $\begin{array}{l}\text { Richard Morgan } \\
\text { USA }\end{array}$ & $\begin{array}{l}\text { Manouchehr Nakhjavani } \\
\text { Iran }\end{array}$ & $\begin{array}{l}\text { Amanda Ode } \\
\text { Sweden }\end{array}$ \\
\hline $\begin{array}{l}\text { Don Morris } \\
\text { Canada }\end{array}$ & $\begin{array}{l}\text { Marek Nalos } \\
\text { Australia }\end{array}$ & $\begin{array}{l}\text { Pardo Oe } \\
\text { UK }\end{array}$ \\
\hline $\begin{array}{l}\text { Giovanni Morrone } \\
\text { Italy }\end{array}$ & $\begin{array}{l}\text { Norbert Nass } \\
\text { Germany }\end{array}$ & $\begin{array}{l}\text { Mitsuru Ohishi } \\
\text { Japan }\end{array}$ \\
\hline $\begin{array}{l}\text { Mohamed Morsy } \\
\text { Saudi Arabia }\end{array}$ & $\begin{array}{l}\text { Pier Giorgio Natali } \\
\text { Italy }\end{array}$ & $\begin{array}{l}\text { Shigeo Ohta } \\
\text { Japan }\end{array}$ \\
\hline $\begin{array}{l}\text { Seyed Javad Mowla } \\
\text { Iran }\end{array}$ & $\begin{array}{l}\text { Urs Nater } \\
\text { Germany }\end{array}$ & $\begin{array}{l}\text { Masato Okamoto } \\
\text { Japan }\end{array}$ \\
\hline $\begin{array}{l}\text { Arijit Mukhopadhyay } \\
\text { India }\end{array}$ & $\begin{array}{l}\text { Pilar Navarro } \\
\text { Spain }\end{array}$ & $\begin{array}{l}\text { H. Okazawa } \\
\text { Japan }\end{array}$ \\
\hline $\begin{array}{l}\text { Debabrata Mukhopadhyay } \\
\text { USA }\end{array}$ & $\begin{array}{l}\text { Marcos Mônico Neto } \\
\text { USA }\end{array}$ & $\begin{array}{l}\text { José M. Olmos } \\
\text { Spain }\end{array}$ \\
\hline $\begin{array}{l}\text { Yosuke Mukoyama } \\
\text { USA }\end{array}$ & $\begin{array}{l}\text { Qimin Ng } \\
\text { USA }\end{array}$ & $\begin{array}{l}\text { Oluwadayo Oluwadara } \\
\text { USA }\end{array}$ \\
\hline $\begin{array}{l}\text { Dafne Müller } \\
\text { Germany }\end{array}$ & $\begin{array}{l}\text { Robert Nibbs } \\
\text { UK }\end{array}$ & $\begin{array}{l}\text { Petr Ostadal } \\
\text { Czech Republic }\end{array}$ \\
\hline $\begin{array}{l}\text { Carine Munaut } \\
\text { Belgium }\end{array}$ & $\begin{array}{l}\text { Michael Nicholson } \\
\text { UK }\end{array}$ & $\begin{array}{l}\text { Arne Ostman } \\
\text { Sweden }\end{array}$ \\
\hline $\begin{array}{l}\text { Susan Murphy } \\
\text { USA }\end{array}$ & $\begin{array}{l}\text { Amelia Nieto } \\
\text { Spain }\end{array}$ & $\begin{array}{l}\text { Megan Othus } \\
\text { USA }\end{array}$ \\
\hline $\begin{array}{l}\text { William Murphy } \\
\text { USA }\end{array}$ & $\begin{array}{l}\text { Hataikarn Nimitphong } \\
\text { Thailand }\end{array}$ & $\begin{array}{l}\text { Christian Ottensmeier } \\
\text { UK }\end{array}$ \\
\hline $\begin{array}{l}\text { Teemu Murtola } \\
\text { Finland }\end{array}$ & $\begin{array}{l}\text { Paola Nistico } \\
\text { Italy }\end{array}$ & $\begin{array}{l}\text { Teresa Otto } \\
\text { Germany }\end{array}$ \\
\hline $\begin{array}{l}\text { Saminathan Muthusamy } \\
\text { USA }\end{array}$ & $\begin{array}{l}\text { Zhaoshan Niu } \\
\text { China }\end{array}$ & $\begin{array}{l}\text { C. Keith Ozaki } \\
\text { USA }\end{array}$ \\
\hline $\begin{array}{l}\text { Seyed Mohammad Nabavi } \\
\text { Iran }\end{array}$ & $\begin{array}{l}\text { Paul Norris } \\
\text { USA }\end{array}$ & $\begin{array}{l}\text { Utpal Pal } \\
\text { USA }\end{array}$ \\
\hline $\begin{array}{l}\text { Takeshi Nabe } \\
\text { Japan }\end{array}$ & $\begin{array}{l}\text { Andrew Norris } \\
\text { USA }\end{array}$ & $\begin{array}{l}\text { Nallasivam Palanisamy } \\
\text { USA }\end{array}$ \\
\hline $\begin{array}{l}\text { Atsushi Nagai } \\
\text { Japan }\end{array}$ & $\begin{array}{l}\text { Mauro Novelli } \\
\text { Italy }\end{array}$ & $\begin{array}{l}\text { Michael Palladino } \\
\text { USA }\end{array}$ \\
\hline
\end{tabular}




\begin{tabular}{|c|c|c|}
\hline $\begin{array}{l}\text { Eleonora Palma } \\
\text { Italy }\end{array}$ & $\begin{array}{l}\text { Sven Perner } \\
\text { Germany }\end{array}$ & $\begin{array}{l}\text { Maurizio Provenzano } \\
\text { Switzerland }\end{array}$ \\
\hline Gaby Palmer & Carlo Perricone & Igor Prudovsky \\
\hline Switzerland & Italy & Russian Federation \\
\hline Giuseppe Palmieri & Michael Peters & Ioannis Psallidas \\
\hline Italy & UK & UK \\
\hline Xinmin Pan & Bryon Petersen & Michele Purrello \\
\hline China & USA & Italy \\
\hline Sandhya Panch & Annacarmen Petrizzo & Alessandro Puzziello \\
\hline USA & Italy & Italy \\
\hline Tanmay Panchabhai & Raffaele Pezzilli & Hui Qian \\
\hline USA & Italy & China \\
\hline Jong Hoon Park & Matthew Phillips & Fabiana Quaglia \\
\hline South Korea & USA & Italy \\
\hline Jiyoung Park & Giulia Piaggio & Mohammed Quttainah \\
\hline South Korea & Italy & Saudi Arabia \\
\hline Mr. Parkhurst & Massimo Pietropaolo & Monte Radeke \\
\hline USA & USA & USA \\
\hline Paola Parronchi & Valeria Pietropaolo & Gajendra Raghava \\
\hline Italy & Italy & India \\
\hline Rosa Maria Pascale & Somchai Pinlaor & Afsar Rahbar \\
\hline Italy & Thailand & Sweden \\
\hline Shibani Pati & Karina Pino-Lagos & Natalia Ramirez \\
\hline USA & Chile & Spain \\
\hline Bhushan Patwardhan & Jalil Pirayesh Islamian & Famela Ramos \\
\hline India & Iran & USA \\
\hline Andreas Paul & Anna Planas & Kanishka Ratnayaka \\
\hline Germany & Spain & USA \\
\hline Kajsa Maria Paulsson & Martin Playford & Arnab Ray \\
\hline Sweden & USA & USA \\
\hline Henrik D. Pedersen & Miroslav Pohanka & Elrashdy Redwan \\
\hline Denmark & Czech Republic & Saudi Arabia \\
\hline Xue Tao Pei & Raimo Pohjanvirta & Kerstin Reimers \\
\hline China & Finland & Germany \\
\hline Maria Marjorette Pena & Katherine Ponder & Jiaqiang Ren \\
\hline USA & USA & USA \\
\hline Dao-Quan Peng & Zoltán Pós & Anna Lena Ress \\
\hline China & Hungary & Austria \\
\hline Cheng-Yuan Peng & Michael Postow & Habtom Ressom \\
\hline Taiwan, China & USA & USA \\
\hline Arianne Perez Garcia & David Pride & Muhammad Riaz \\
\hline USA & USA & Pakistan \\
\hline
\end{tabular}




\begin{tabular}{|c|c|c|}
\hline $\begin{array}{l}\text { Andrea Ribeiro-Dos-Santos } \\
\text { Brazil }\end{array}$ & $\begin{array}{l}\text { Paolo Ruggiero } \\
\text { Italy }\end{array}$ & $\begin{array}{l}\text { Anna Sapino } \\
\text { Italy }\end{array}$ \\
\hline $\begin{array}{l}\text { Biagio Ricciuti } \\
\text { Italy }\end{array}$ & $\begin{array}{l}\text { Pekka Ruotsalainen } \\
\text { Finland }\end{array}$ & $\begin{array}{l}\text { Shantanu Sapru } \\
\text { India }\end{array}$ \\
\hline $\begin{array}{l}\text { Ann Richmond } \\
\text { USA }\end{array}$ & $\begin{array}{l}\text { Julie Russier } \\
\text { France }\end{array}$ & $\begin{array}{l}\text { Francesca Sarlo } \\
\text { Italy }\end{array}$ \\
\hline $\begin{array}{l}\text { Neil Riordan } \\
\text { Panama }\end{array}$ & $\begin{array}{l}\text { Sergio Rutella } \\
\text { Qatar }\end{array}$ & $\begin{array}{l}\text { Manoj Sarma } \\
\text { USA }\end{array}$ \\
\hline $\begin{array}{l}\text { Loris Rizzello } \\
\text { UK }\end{array}$ & $\begin{array}{l}\text { Sergio Rutella } \\
\text { Qatar }\end{array}$ & $\begin{array}{l}\text { Seetharama Sastry Konduru } \\
\text { Qatar }\end{array}$ \\
\hline $\begin{array}{l}\text { Lidia Robert } \\
\text { USA }\end{array}$ & $\begin{array}{l}\text { Carrie Ruxton } \\
\text { UK }\end{array}$ & $\begin{array}{l}\text { Jean Frederic Sauniere } \\
\text { France }\end{array}$ \\
\hline $\begin{array}{l}\text { Anna Rocco } \\
\text { Italy }\end{array}$ & $\begin{array}{l}\text { Ogino S. } \\
\text { USA }\end{array}$ & $\begin{array}{l}\text { Nazish Sayed } \\
\text { USA }\end{array}$ \\
\hline $\begin{array}{l}\text { Rafael Rocha } \\
\text { Brazil }\end{array}$ & $\begin{array}{l}\text { Bashar Saad } \\
\text { Palestinian Territory }\end{array}$ & $\begin{array}{l}\text { Giorgio Scagliotti } \\
\text { Italy }\end{array}$ \\
\hline $\begin{array}{l}\text { Ramón Rodrigo } \\
\text { Chile }\end{array}$ & $\begin{array}{l}\text { Marianna Sabatino } \\
\text { USA }\end{array}$ & $\begin{array}{l}\text { Richard Schaefer } \\
\text { Germany }\end{array}$ \\
\hline $\begin{array}{l}\text { Rosalia Rodriguez-Rodriguez } \\
\text { Spain }\end{array}$ & $\begin{array}{l}\text { Ulrich Sack } \\
\text { Germany }\end{array}$ & $\begin{array}{l}\text { Francesco Paolo Schena } \\
\text { Italy }\end{array}$ \\
\hline $\begin{array}{l}\text { Howard Roemer } \\
\text { USA }\end{array}$ & $\begin{array}{l}\text { Sakthivel Sadayappan } \\
\text { USA }\end{array}$ & $\begin{array}{l}\text { Howard Scher } \\
\text { USA }\end{array}$ \\
\hline $\begin{array}{l}\text { Michael Rogers } \\
\text { USA }\end{array}$ & $\begin{array}{l}\text { Sona Saksena } \\
\text { USA }\end{array}$ & $\begin{array}{l}\text { Sophia E. Schiza } \\
\text { Greece }\end{array}$ \\
\hline $\begin{array}{l}\text { Andreas Roidl } \\
\text { Germany }\end{array}$ & $\begin{array}{l}\text { Gianluca Sala } \\
\text { Italy }\end{array}$ & $\begin{array}{l}\text { Erwin Schleicher } \\
\text { Germany }\end{array}$ \\
\hline $\begin{array}{l}\text { Francisco Romero } \\
\text { Spain }\end{array}$ & $\begin{array}{l}\text { Athanasios Salifoglou } \\
\text { Greece }\end{array}$ & $\begin{array}{l}\text { Dorit Schleinitz } \\
\text { Germany }\end{array}$ \\
\hline $\begin{array}{l}\text { Dario Ronchi } \\
\text { Italy }\end{array}$ & $\begin{array}{l}\text { Gil Salles } \\
\text { Brazil }\end{array}$ & $\begin{array}{l}\text { Antje Schmidt } \\
\text { Germany }\end{array}$ \\
\hline $\begin{array}{l}\text { Guanghua Rong } \\
\text { China }\end{array}$ & $\begin{array}{l}\text { Goli Samimi } \\
\text { USA }\end{array}$ & $\begin{array}{l}\text { Gerold Schuler } \\
\text { Germany }\end{array}$ \\
\hline $\begin{array}{l}\text { Ronald Rooke } \\
\text { France }\end{array}$ & $\begin{array}{l}\text { Marta Sanchez-Carbayo } \\
\text { Spain }\end{array}$ & $\begin{array}{l}\text { Sandra Schüssler } \\
\text { Austria }\end{array}$ \\
\hline $\begin{array}{l}\text { Margaret Ross } \\
\text { USA }\end{array}$ & $\begin{array}{l}\text { Pau Sancho-Bru } \\
\text { Spain }\end{array}$ & $\begin{array}{l}\text { Clare Scott } \\
\text { Australia }\end{array}$ \\
\hline $\begin{array}{l}\text { Giulio Rossi } \\
\text { Italy }\end{array}$ & $\begin{array}{l}\text { Francesca Santoni De Sio } \\
\text { Italy }\end{array}$ & $\begin{array}{l}\text { Cristian Secchi } \\
\text { Italy }\end{array}$ \\
\hline $\begin{array}{l}\text { Claudia Rossig } \\
\text { Germany }\end{array}$ & $\begin{array}{l}\text { Ney Santos } \\
\text { Brazil }\end{array}$ & $\begin{array}{l}\text { Silvia Selleri } \\
\text { Canada }\end{array}$ \\
\hline $\begin{array}{l}\text { Susanta Roychoudhury } \\
\text { India }\end{array}$ & $\begin{array}{l}\text { Gaetano Santulli } \\
\text { USA }\end{array}$ & $\begin{array}{l}\text { Carlo Selmi } \\
\text { USA }\end{array}$ \\
\hline
\end{tabular}




\begin{tabular}{|c|c|c|}
\hline $\begin{array}{l}\text { Lorenzo Sempere } \\
\text { USA }\end{array}$ & $\begin{array}{l}\text { Lin Shi } \\
\text { China }\end{array}$ & $\begin{array}{l}\text { Joyce Solheim } \\
\text { USA }\end{array}$ \\
\hline $\begin{array}{l}\text { Aritro Sen } \\
\text { USA }\end{array}$ & $\begin{array}{l}\text { Ning Shi } \\
\text { USA }\end{array}$ & $\begin{array}{l}\text { David Solomon } \\
\text { USA }\end{array}$ \\
\hline $\begin{array}{l}\text { Nabonita Sengupta } \\
\text { India }\end{array}$ & $\begin{array}{l}\text { Takatsune Shimizu } \\
\text { Japan }\end{array}$ & $\begin{array}{l}\text { Mark Soloski } \\
\text { USA }\end{array}$ \\
\hline $\begin{array}{l}\text { Sung Wook Seo } \\
\text { USA }\end{array}$ & $\begin{array}{l}\text { Yoshihiro Shirai } \\
\text { Japan }\end{array}$ & $\begin{array}{l}\text { Michele Sommariva } \\
\text { Italy }\end{array}$ \\
\hline $\begin{array}{l}\text { Naresh Babu V. Sepuri } \\
\text { India }\end{array}$ & $\begin{array}{l}\text { Yehuda Shoenfeld } \\
\text { Israel }\end{array}$ & $\begin{array}{l}\text { Deok-Soo Son } \\
\text { USA }\end{array}$ \\
\hline $\begin{array}{l}\text { Suraj Serai } \\
\text { USA }\end{array}$ & $\begin{array}{l}\text { Emma Shtivelman } \\
\text { USA }\end{array}$ & $\begin{array}{l}\text { Avinash Sonawane } \\
\text { India }\end{array}$ \\
\hline $\begin{array}{l}\text { Natalya Seredkina } \\
\text { Norway }\end{array}$ & $\begin{array}{l}\text { Thomas Shupe } \\
\text { USA }\end{array}$ & $\begin{array}{l}\text { Libing Song } \\
\text { China }\end{array}$ \\
\hline $\begin{array}{l}\text { Feride Severcan } \\
\text { Turkey }\end{array}$ & $\begin{array}{l}\text { Bruno Silva-Santos } \\
\text { Portugal }\end{array}$ & $\begin{array}{l}\text { Koh-Hei Sonoda } \\
\text { Japan }\end{array}$ \\
\hline $\begin{array}{l}\text { Patricia Severino } \\
\text { Brazil }\end{array}$ & $\begin{array}{l}\text { Charles Simone } \\
\text { USA }\end{array}$ & $\begin{array}{l}\text { Claudio Sorio } \\
\text { Italy }\end{array}$ \\
\hline $\begin{array}{l}\text { Ioannis Sgouralis } \\
\text { USA }\end{array}$ & $\begin{array}{l}\text { James Simpkins } \\
\text { USA }\end{array}$ & $\begin{array}{l}\text { Alexander Sorokin } \\
\text { USA }\end{array}$ \\
\hline $\begin{array}{l}\text { Ramraj Sh. } \\
\text { USA }\end{array}$ & $\begin{array}{l}\text { Peter Sims } \\
\text { USA }\end{array}$ & $\begin{array}{l}\text { Vinicius Sortica } \\
\text { Brazil }\end{array}$ \\
\hline $\begin{array}{l}\text { Baoen Shan } \\
\text { China }\end{array}$ & $\begin{array}{l}\text { Virendra Singh } \\
\text { India }\end{array}$ & $\begin{array}{l}\text { John Souglakos } \\
\text { Greece }\end{array}$ \\
\hline $\begin{array}{l}\text { Jingxuan Shan } \\
\text { Qatar }\end{array}$ & $\begin{array}{l}\text { Anup Singh } \\
\text { India }\end{array}$ & $\begin{array}{l}\text { Andrew South } \\
\text { USA }\end{array}$ \\
\hline $\begin{array}{l}\text { E. Sharples } \\
\text { UK }\end{array}$ & $\begin{array}{l}\text { Ritesh Singh } \\
\text { India }\end{array}$ & $\begin{array}{l}\text { Claudio Spada } \\
\text { Italy }\end{array}$ \\
\hline $\begin{array}{l}\text { Yuehwei Shen } \\
\text { USA }\end{array}$ & $\begin{array}{l}\text { Arvind Singla } \\
\text { Canada }\end{array}$ & $\begin{array}{l}\text { Giulio Spagnoli } \\
\text { Switzerland }\end{array}$ \\
\hline $\begin{array}{l}\text { Rui Sheng } \\
\text { China }\end{array}$ & $\begin{array}{l}\text { Sridhar Sivasubbu } \\
\text { India }\end{array}$ & $\begin{array}{l}\text { Christoph Spiess } \\
\text { USA }\end{array}$ \\
\hline $\begin{array}{l}\text { Natalie Shenker } \\
\text { UK }\end{array}$ & $\begin{array}{l}\text { Tomas Skripcak } \\
\text { Germany }\end{array}$ & $\begin{array}{l}\text { Neil J. Spratt } \\
\text { Australia }\end{array}$ \\
\hline $\begin{array}{l}\text { Hilary Sheppard } \\
\text { New Zealand }\end{array}$ & $\begin{array}{l}\text { Philipp Skroblin } \\
\text { UK }\end{array}$ & $\begin{array}{l}\text { Ralf Sprengers } \\
\text { The Netherlands }\end{array}$ \\
\hline $\begin{array}{l}\text { Warren Sherman } \\
\text { Belgium }\end{array}$ & $\begin{array}{l}\text { Nicoline Smit } \\
\text { The Netherlands }\end{array}$ & $\begin{array}{l}\text { Danielle Springer } \\
\text { USA }\end{array}$ \\
\hline $\begin{array}{l}\text { Yongguo Shi } \\
\text { China }\end{array}$ & $\begin{array}{l}\text { Maarten Snoeijs } \\
\text { The Netherlands }\end{array}$ & $\begin{array}{l}\text { Randall Stafford } \\
\text { Canada }\end{array}$ \\
\hline $\begin{array}{l}\text { Ruihua Shi } \\
\text { China }\end{array}$ & $\begin{array}{l}\text { Robert Sobol } \\
\text { USA }\end{array}$ & $\begin{array}{l}\text { Robert Stahelin } \\
\text { USA }\end{array}$ \\
\hline
\end{tabular}


Ivan Stanojevic

Serbia

Elena Stansky

USA

Ted Steiner

Canada

Marta Stelmach-Mardas

Germany

Verena Stiegelbauer

Austria

Georg Stoecklin

Germany

Karen Stokes

USA

Jan Stolk

The Netherlands

Areti Strati

Greece

Raphael Stricker

USA

Patrick Stuart

USA

Christina Stuelten

USA

Ali Sultan
USA

Shubhankar Suman

USA

Ming Sun

China

Huijun Sun

China

Tao Sun

USA

Yang Sun

USA

Inge Marie Svane

Denmark

Abhisek Swaika

USA

Wing-Kin Syn

UK
Sébastien Tabariès

Canada

Frank Tacke

Germany

Cliff Taggart

UK

Maria Tagliamonte Italy

Hiroshi Takemori

Japan

Daryush Talei

Iran

Giuseppe Tambussi

Italy

Yi Tan

USA

Eng M. Tan

USA

Xianglin Tan

USA

Takuji Tanaka

Japan

Halil Tanboga

Turkey

Qionglan Tang

China

Jing Tang

China

Jianming Tang

USA

Yaoliang Tang

USA

Magdalena Tary-Lehmann

USA

Francesca Tavano

Italy

Kathryn Taylor

UK

Heather Teague

USA

Mohamed-Ramzi Temanni

Qatar
Anja Ten Brinke

The Netherlands

Benno Ter Kuile

The Netherlands

Yasuo Terauchi

Japan

Luigi Terracciano

Switzerland

Paula Terraciano

Brazil

Annalisa Terranegra

Italy

Annalisa Terranegra

Qatar

Osamu Tetsu

USA

Shikha Tewari

India

Charles Theuer

USA

Susan Thibeault

USA

Gaetano Thiene

Italy

Victor Ljl Thijssen

The Netherlands

Albert Thomas

USA

M. Albert Thomas

USA

Bolaji Thomas

USA

Tanushree Thote

USA

Weidong Tian

China

Jozsef Timar

Hungary

Vincent Timmerman

Belgium

Antony Tin

USA 
Marianne Tinguely

Switzerland

Gabriele Toietta

Italy

Maria Lauda Tomasi

USA

Sara Tomei

Qatar

Maja Tomicic

Germany

Ikuo Tooyama

Japan

Maria Lina Tornesello

Italy

Gregory Tranah

USA

Gabriela Trevisan

Brazil

Hui-Ju Tsai

Taiwan, China

John Tsang

USA

Marco Tucci

Italy

Paul Tumeh

USA

Tao-Hsin Tung

Taiwan

Heth Turnquist

USA

Cameron Turtle

USA

Sandra Tuyaerts

Belgium

Argyris Tzouvelekis

Greece

Naoto Ueno

USA

Lena Uller

Sweden

Cuneyt Ulutin

Turkey
Florian Unger

Germany

Masuko Ushio-Fukai

USA

Pascal Vachon

Canada

Silvia Vai

Italy

Mariapia Vairetti

Italy

Sergey Y. Vakhrushev

Denmark

Soraya Valles

Spain

Johan Van Der Vlag

The Netherlands

Eric Van Dyck

Luxembourg

Mireille Van Gele

Belgium

Arthur Vandenbark

USA

Moriel Vandsburger

USA

Venkat Vangaveti

Australia

Carlos Vasconcelos

Portugal

Roger Vaughan

USA

Florence Velge-Roussel

France

Paulo Verardi

USA

Mohamud Verjee

Qatar

Suresh Verma

USA

Gianpaolo Vidili

Italy

Regina Maria Vilela

Brazil
Sandhya Visweswariah

India

Flavia Vitale

USA

Santiago Viteri

Spain

Isabel Vogel

France

Barbara Vona

Germany

Agarwal Vr.

India

Lukas Vrba

USA

Krishna Vyas

USA

Ori Wald

Israel

David Walker

Austria

Judd Walson

USA

Yu-Jui Wan

USA

Hans Wandall

Denmark

Yingwei Wang

China

Haiyun Wang

China

Anhui Wang

China

Xin Wang

China

Shukui Wang

China

De-Shen Wang

China

Jun Wang

China

Feng-Sheng Wang

Taiwan 
Shih-Wei Wang

Taiwan

Weiguang Wang

UK

I-Ming Wang

USA

Zhaoming Wang

USA

Chunling Wang

China

Rui Wang-Sattler

Germany

Yoo Wan-Hee

South Korea

Kelly Warfield

USA

David G. Watt

UK

Jeffrey Weber

USA

Barbara Wegiel

USA

Qize Wei

USA

George Weiner

USA

Herschel Weintraub

USA

James Wells

Australia

James Welsh

USA

Tom Van Wezel

The Netherlands

Nicole White

USA

Matt Whiteman

UK

Robert Wilensky

USA

Karen Willard-Gallo

Belgium
Jed Wiltzius

USA

Johann Wojta

Austria

Chris Wong

China

Thian-Sze Wong

Hong Kong

Wing Tak Jack Wong

USA

Haijing Wu

China

Yingli Wu

China

Weizhong Wu

China

Wen-Bin Wu

Taiwan

Reen Wu

USA

Xiaobo Wu

USA

Chia-Chao Wu

Taiwan

Vegard Bruun Wyller

Norway

Mo X.

China

Zhidao Xia

UK

Andy Xiang

China

Zhengwen Xiao

Canada

Hang Xiao

China

G. Xie

China

Baoling Xing

China

Feng Xu

China
Rui-Hua Xu

China

Wenrong Xu

China

Feng Xu

China

Sushma Yadav

USA

Feng Yan

China

Jun Yan

China

Maria Yanez-Mo

Spain

Qinglin Yang

China

Zhixiong Yang

China

Cheng Yang

China

Kejian Yang

USA

Wassana Yantasee

USA

Jiayi Yao

China

Ming Yao

China

Kosei Yasumoto

Japan

Dongqing Ye

China

Shoudong Ye

China

Yao-Tsung Yeh

Taiwan

Samantha Yeligar

USA

Pen-Hui Yin

Taiwan

Song Yongmei

China 
Yukie Yoshii

Japan

Seungkwon You

South Korea

Jianxiu Yu

China

Tao Yu

China

Hao Yu

USA

Jean-François Zagury

France

Marco Zarbin

USA

Paul Zarogoulidis

Greece

Amir Zarrinpar

USA

Flora Zavala

France

\section{Abdel-Rahman Zekri}

Egypt

Published online: 11 March 2016
Davide Zella

USA

Dimitrios Zeugolis

Ireland

Shu Zhang

China

He Zhang

China

Shenghui Zhang

China

Di Zhang

China

Haitao Zhao

China

Ming Zhao

China

Dejian Zhao

China

Minghui Zhao

China
Junhua Zheng

China

Fei Zhou

China

Yunfeng Zhou

China

J. Zhou

China

Xi Kathy Zhou

USA

Dengna Zhu

China

Ping Zhu

China

Shaobo Zhu

USA

David Zidar

USA

Anna Linda Zignego

Italy
Submit your next manuscript to BioMed Central and we will help you at every step:

- We accept pre-submission inquiries

- Our selector tool helps you to find the most relevant journal

- We provide round the clock customer support

- Convenient online submission

- Thorough peer review

- Inclusion in PubMed and all major indexing services

- Maximum visibility for your research

Submit your manuscript at www.biomedcentral.com/submit 